\section{Ingeniería}

CONTENIDO

Artículos

Análisis numérico de un sistema de aire acondicionado empleando
mallas estructuradas y no estructurades A

Funciones de regresión para caudales extremos en la Vertiente Rojas, Nazareth; Aguilar, José Francisco; Solís, Hernán. Evaluación de daños por agresión ambiental en viviendas
de concreto reforzado. Solís, Rómel; Moreno, Eric; Jiménez, Felipe; Rosas, Victorino.

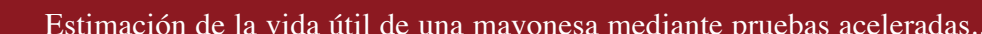
Estimación de la vida útil de una mayonesa
García, Claudia; Molina, Manuel.

Inestabilidad oscilatoria de tensión debido a los motores de inducción …….....................65-75 rde, Gustavo; Araya, Eddie.

Estabilidad y colapso de tensión en sistemas eléctricos Araya, Eddie.

Obtención de pulpa a la soda antraquinona de Tectona grandis creciendo en Costa Rica...........................

Protocolo evaluador para el manejo de desechos y la seguridad ocupacional
aplicado al laboratorio clínico del Hospital San Francisco de Asís de Grecia. Ruiz, Francisco.

9. Euler: su contex
Vargas, Celso.

10. Reserva de resistencia de edificios porticados de concreto armado disenaados conforme al ACI-318/ IBC 2006 ........ Análisis y comentarios

Herrera, Rodolfo.

breve semblanza.

Trabajos de Graduación 2007

Lista de proyectos de graduación de grado y posgrado

Normas

$\sum_{\text {EDTORAL }}$

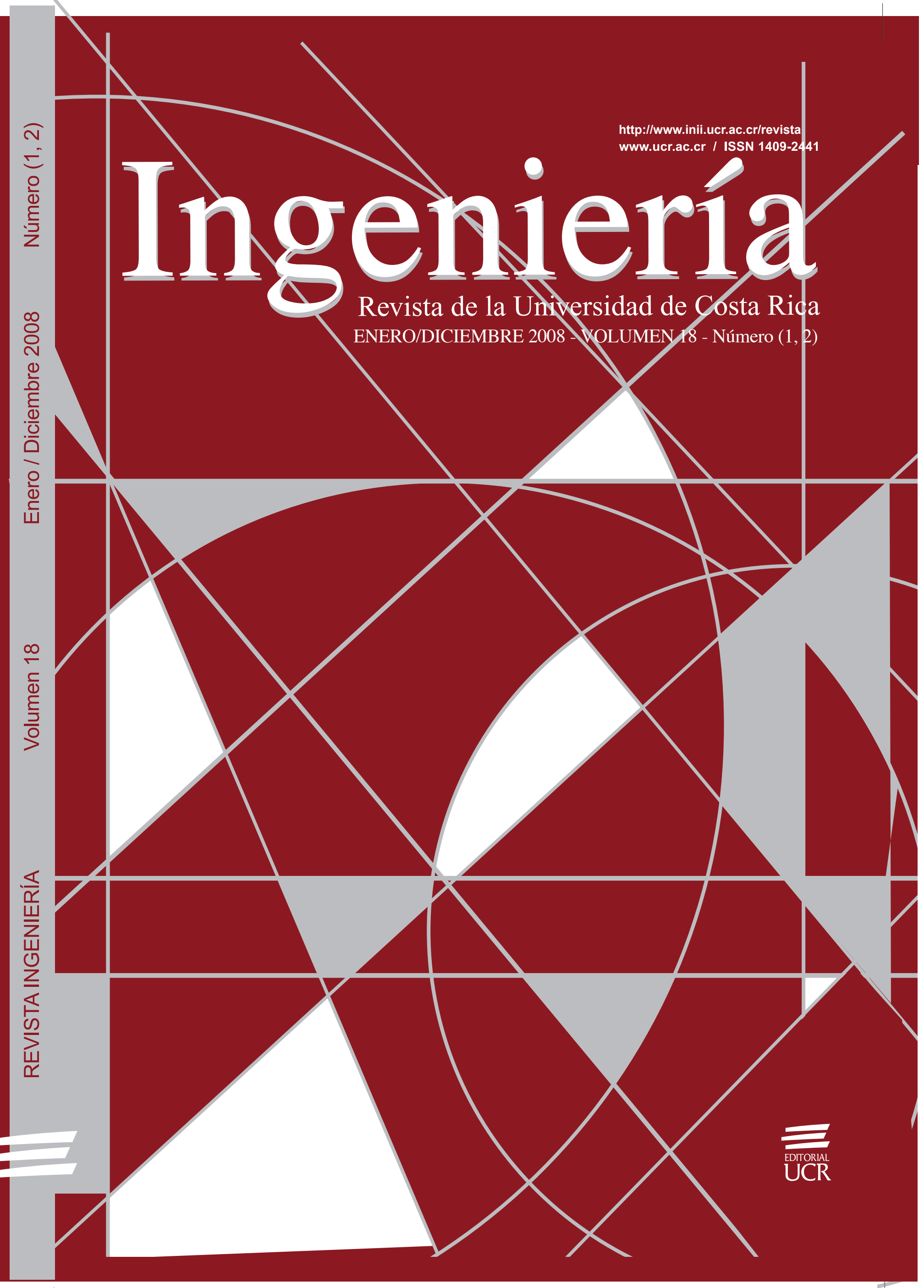




\title{
OBTENCIÓN DE PULPA A LA SODA ANTRAQUINONA DE Tectona grandis CRECIENDO EN COSTA RICA
}

\begin{abstract}
Resumen
Se obtuvo pulpa por el método soda antraquinona a partir de Teca (Tectona grandis) de seis años, proveniente de una plantación de la zona de San Carlos, Alajuela.

Para ello se realizó un diseño estadístico factorial con dos niveles y tres variables: la temperatura de cocción, el tiempo de cocción y la concentración de hidróxido de sodio en porcentaje másico; como variables fijas se establecieron la relación de baño (1/6), la cantidad de madera seca inicial (140,0 g) y la concentración de antraquinona en porcentaje másico $(0,1 \%)$, siendo las variables de respuesta el rendimiento másico y el contenido de lignina en la pulpa (ambos porcentajes en base seca).

Los mejores resultados se obtuvieron a una temperatura de $160{ }^{\circ} \mathrm{C}$, un tiempo de cocción de 45 min, y una concentración de hidróxido de sodio del $7 \%$, con un rendimiento másico de pulpa de 45,5 \% y una deslignificación de $98 \%$. El tiempo fue más corto que el empleado en otras investigaciones similares, y la temperatura y la concentración de hidróxido de sodio, a pesar de ser bajas, podrían disminuirse de acuerdo con los resultados del estudio.

La pulpa obtenida fue de color beige claro y sin astillas remanentes; el pulpeo soda-antraquinona fue efectivo para la cocción de la madera y para la producción de la pulpa, muy selectivo en la eliminación de la lignina sin deterioro de la celulosa, lo que se refleja en un rendimiento alto.
\end{abstract}

Palabras clave: teca, Tectona grandis, antraquinona, soda, pulpa

\begin{abstract}
A soda-anthraquinone process was used to obtain pulp from six year old Teak (Tectona grandis) wood, farm grown in northern Costa Rica (San Carlos, Alajuela).

The experiment used statistical factorial design with two levels and three variables. The variables were cooking time, cooking temperature and sodium hydroxide concentration in mass percentage; the fixed variables were bathing relationship (1/6), initial quantity of dry wood $(140,0 \mathrm{~g})$ and anthraquinone concentration in mass percentage $(0,1 \%)$. Answer variables were mass yield and pulp lignin content (both on dry base percentages).

Best results were obtained at a temperature of $160^{\circ} \mathrm{C}$, cooking time of $45 \mathrm{~min}$, and sodium hydroxide concentration of $7 \%$ with a pulp mass yield of $45,5 \%$ and delignification of $98 \%$. This time is shorter than that used in similar research and the temperature and sodium hydroxide percentage, despite being low, can be diminished further according to the results.

The pulp was light beige, and showed no remaining chips or splinters; therefore, it can be stated that the sodaanthraquinone pulping was effective for wood cooking and pulp production, since it is very selective eliminating lignin without harming the cellulose, which gives a high yield.
\end{abstract}

Key words: teak, Tectona grandis, anthraquinone, soda, pulp.

Recibido: 09 de febrero del 2009 • Aprobado: 17 de abril del 2009

\section{INTRODUCCIÓN}

Debido al interés creciente por conservar los recursos naturales y lograr la armonía entre las industrias y la naturaleza, a nivel mundial han surgido movimientos e iniciativas que buscan aumentar el área de las plantaciones de madera amigadas con el ambiente, así como introducir el conocimientonecesario paraqueestas seconviertan en la más importante fuente de madera de alta calidad que en el futuro satisfaga completamente los mercados locales e internacionales. 
Estas iniciativas han apuntado principalmente hacia la plantación de teca (Tectona grandis), debido a que es una especie maderable de crecimiento rápido, que ha llegado a constituirse en la especie más importante de las plantaciones de madera de alta calidad presentes en el trópico. Dentro de estas iniciativas se encuentra TECA 2000, la cual busca involucrar al sector privado, gobierno, comunidades locales y grupos ambientalistas y brindar el soporte (financiero y técnico) que necesiten los productores para obtener más madera y de mejor calidad, de una manera social y ambientalmente aceptables. Este proyecto busca el establecimiento de un millón y medio a dos millones de hectáreas de teca, en menos de cuarenta años para eliminar la presión sobre los bosques naturales y lograr suplir la demanda de madera de calidad, produciendo al menos dieciséis millones de metros cúbicos por año. En Costa Rica durante varios años se desarrollaron programas de reforestación que introdujeron la teca, la cual representa un buen porcentaje de estos programas y de las plantaciones con que actualmente cuenta el país.

El descubrimiento de la antraquinona como aditivo en el proceso Kraft, ha abierto nuevas oportunidades tecnológicas. La antraquinona es un polvo amarillento con fórmula molecular $\mathrm{C}_{14} \mathrm{H}_{8} \mathrm{O}_{2}$, de peso molecular igual a 208,23 $\mathrm{g} / \mathrm{gmol}$, con una densidad de $1,438 \mathrm{~g} / \mathrm{cm}^{3} \mathrm{y}$ temperaturas de fusión y ebullición de $286{ }^{\circ} \mathrm{C}$ y $378,8{ }^{\circ} \mathrm{C}$ respectivamente. Resultado de un número creciente de estudios, la antraquinona es considerada el primer aditivo realmente eficaz y de valor práctico, tanto a nivel industrial como comercial en procesos alcalinos. La antraquinona causa una oxidación del grupo reductor terminal de los carbohidratos, generando una estabilidad en cuanto a las reacciones de despolimerización terminal. Dicha estabilización resulta en proteger los carbohidratos contra las reacciones de degradación y consecuentemente, un aumento en el rendimiento. Por otra parte, la antraquinona causa sobre la lignina una aceleración de las reacciones de hidrólisis formando fragmentos de lignina de menor peso molecular, lo que se refleja en un aumento en la tasa de deslignificación (Gomes da Silva, 1994).
La adición de antraquinona al licor de producción a la sosa en cantidades tan reducidas como $0,05 \%$ a $0,10 \%$ de la madera producirá velocidades de deslignificación, rendimientos de pulpa y propiedades en cuanto a la resistencia, análogas a las que se obtienen mediante la producción Kraft. Puede también agregarse la antraquinona a los licores de digestión Kraft para lograr mejorías tanto en el rendimiento como en las propiedades de la pulpa (Casey, 1990).

En los procesos comerciales siempre queda lignina residual, para su eliminación habría que extender los tiempos de cocción, provocando que los rendimientos bajaran, junto con una baja selectividad en la deslignificación y pulpas de menos calidad (Manji, 1996).

\section{METODOLOGÍA}

La materia prima utilizada y analizada fue extraída de Altamira de Aguas Zarcas de San Carlos, Alajuela; de la empresa Flor y Fauna S. A., del proyecto TEAKWOOD V, el cual se localiza entre las coordenadas horizontales 494-497 y verticales 283-284 de la hoja cartográfica Tres Amigos número 3347 IV. Esta zona pertenece a la unidad biótica tropical, tropical húmeda con uno o dos meses secos. El proyecto presenta un bosque tropical lluvioso de bajura, con topografía plana-ondulada y colinas, el clima es húmedo muy caliente, una evapotranspiración anual mayor que $1710 \mathrm{~mm}$. Por información del Instituto Meteorológico Nacional, se registra una precipitación promedio anual de 3 076,7 $\mathrm{mm}$, una temperatura promedio anual de 26,6 ${ }^{\circ} \mathrm{C}$, con una máxima anual de $31,4{ }^{\circ} \mathrm{C}$ y mínima anual de $20,2^{\circ} \mathrm{C}$. Los vientos predominantes en la zona, presentan velocidad promedio anual de $12,3 \mathrm{~km} / \mathrm{h}$ y el terreno presenta pendientes entre un $0 \%$ y $40 \%$ y una elevación de $90 \mathrm{~m}$ a $120 \mathrm{~m}$.

Se trabajó con seis trozas de madera de teca de seis años, las cuales se astillaron en la empresa FIDERICA S. A., ubicada en Río Claro de Golfito utilizando un astillador industrial para celulosa, que según su diseño produce 
astillas de $25,4 \mathrm{~mm} \times 25,4 \mathrm{~mm}$. De vuelta al Laboratorio, las astillas de teca se extendieron en el suelo para eliminar el exceso de humedad que poseían y evitar la degradación del material por pudrición. Se tomaron cuatro muestras de aproximadamente $25 \mathrm{~kg}$ de material húmedo; se tamizaron recogiendo solamente el material que pasaba por una malla de 1 mesh (abertura de $2,54 \mathrm{~cm}$ de diámetro) y que quedaba sobre un tamiz de 10 mesh (abertura de 2,00 mm). Posteriormente se realizó una selección del material recogido en el tamizado para eliminarle materiales extraños como hojas, piedras y secciones de madera o corteza sin astillar. El material tamizado y seleccionado se ubicó en un cuarto a condiciones controladas de $23^{\circ} \mathrm{C}$ y $50 \%$ de humedad relativa.

En secciones transversales del árbol, se determinó el área total ocupada por la albura y por el duramen. Debido a la marcada diferencia visual que existe entre albura y duramen en la teca, utilizando hojas de papel china se copió el contorno de la albura y del duramen; luego se recortaron y por medio de un medidor de área, se determinó el área ocupada por cada parte, en total se analizaron diez secciones transversales. Con los datos obtenidos se calculó del área transversal los valores promedio y la desviación estándar para los porcentajes de albura y de duramen.

Para la determinación de la densidad verde se tomaron muestras de las astillas totales, se pesaron tres muestras de $100 \mathrm{~g}$ con exactitud de $0,1 \mathrm{mg}$ y se registró este como el peso inicial húmedo. Las astillas se sumergieron en agua durante $48 \mathrm{~h}$, luego se dejaron sobre un tamiz durante una hora para eliminar el exceso de agua. En una probeta de $1000 \mathrm{~mL}$ se agregaron poco menos de $650 \mathrm{~mL}$ aforando hasta 650 $\mathrm{mL}$. Se agregaron las astillas en la probeta y se registró el cambio de volumen como volumen verde; se escurrieron nuevamente las astillas y se dejaron en un horno a $103{ }^{\circ} \mathrm{C}$ durante 24 h. Finalmente, se pesaron y se registró el dato como masa final seca y se procedió a calcular el contenido de humedad y el peso específico básico (LATU, 1992).

Se tomaron al azar cien astillas de las seleccionadas para los pulpeos y se les midieron el largo, el ancho y el grosor, utilizando un pie de rey digital, para calcular posteriormente los tamaños promedios de las dimensiones medidas.

\subsection{Pulpeo a la soda antraquinona}

Para determinar la relación líquido/sólido a utilizar en los pulpeos, se colocaron muestras de $25 \mathrm{~g}$ de astillas en seis beakers de $400 \mathrm{~mL}$. Se agregaron volúmenes de $(100 ; 125 ; 150 ; 175$; 200 y 225) $\mathrm{mL}$ de agua, uno en cada beaker. Las astillas se compactaron antes de agregar el agua y se dejaron reposar por $30 \mathrm{~min}$ en el líquido. Se observó luego el grado de humedecimiento, de flotación de las astillas y de cobertura, del líquido. Se escogió la relación que cubría más astillas sin tener mucho líquido por debajo de las astillas cuando estas flotan, para evitar excesos innecesarios ya que el líquido o licor remanente, será un efluente que hay que separar y tratar posteriormente.

Se pulpeó el material por el método soda antraquinona, para ello se realizó un diseño estadístico factorial con dos niveles y tres variables (23). Se utilizaron como variables de diseño la temperatura de cocción $\left(160{ }^{\circ} \mathrm{C}\right.$ y $170{ }^{\circ} \mathrm{C}$ ), el tiempo de cocción (45 min y 75 min) y la concentración de hidróxido de sodio en porcentaje másico (7 \% y $10 \%)$. Como variables fijas se establecieron la relación de baño (1/6), la cantidad de madera seca inicial $(140,0 \mathrm{~g})$ y la concentración de antraquinona en porcentaje másico $(0,1 \%)$. Como variables de respuesta se utilizaron el rendimiento másico y el contenido de lignina en la pulpa (ambos porcentajes en base seca). En el Cuadro 1 se especifican las cantidades utilizadas de reactivos y de materia prima.

Se colocaron $140 \mathrm{~g}$ de astillas de humedad conocida en el reactor, se compactaron en el fondo del mismo y luego se le agregó el licor de cocción correspondiente a la corrida por realizar. El reactor de trabajo se cerró herméticamente para evitar fugas y se inició el calentamiento. Al alcanzarse la temperatura se comenzó a contar el tiempo de cocción; al término del mismo se realizó un enfriamiento súbito del reactor hasta aproximadamente $60{ }^{\circ} \mathrm{C}$. Este procedimiento se utilizó en cada 
Cuadro 1. Cantidad de reactivos y material utilizados para ambos niveles de la concentración de hidróxido de sodio en el diseño.

\begin{tabular}{lcc}
\multicolumn{1}{c}{ Nivel } & $\mathbf{1}$ & $\mathbf{2}$ \\
Concentración de $\mathrm{NaOH}(\%)$ & 7 & 10 \\
Masa seca de astillas $(\mathrm{g})$ & 140,0 & 140,0 \\
Masa hidróxido de sodio $(\mathrm{g})$ & 58,8 & 84,0 \\
Masa de antraquinona $(\mathrm{g})$ & 0,84 & 0,84 \\
Masa de agua fresca $(\mathrm{g})$ & 780,4 & 755,2
\end{tabular}

Fuente: (El autor y la autora).

corrida y correspondió al proceso de cocción únicamente.

Como la mayoría de las investigaciones realizadas con pulpeo a la soda están dirigidas a materiales maderables, las variables temperatura y tiempo de cocción se fijaron tomando como punto de referencia los antecedentes bibliográficos, y a partir de varias pruebas experimentales realizadas. La concentración de antraquinona se fijó siguiendo la conclusión de Rodríguez (2000) sobre el buen desempeño de esta en un proceso de pulpeo similar. El orden de realización de las corridas se estableció al azar y de igual forma se escogió una corrida para repetirla dos veces y poder estimar con ellas la varianza correspondiente del experimento. Para cada una de las corridas se anotó la presión alcanzada, los tiempos de calentamiento y de enfriamiento, el volumen de licor negro obtenido y se recogió una muestra de dicho licor para su posterior análisis.

Después del proceso de cocción, la mezcla dentro del reactor se separó y se midió el volumen del licor recogido; la pulpa se colocó dentro de una bolsa de tela donde se lavó durante 45 min con agua. La pulpa lavada se centrifugó y se le midió la masa húmeda final; de esta se tomaron tres muestras para determinar el contenido de humedad, llevándolas a un horno a $103{ }^{\circ} \mathrm{C}$ hasta sequedad total. Calculando la humedad promedio, se obtuvo la masa seca final y con ésta y la masa seca inicial se calcula el rendimiento másico de cocción para cada corrida.

Para determinar el porcentaje de astillas presentes después de la cocción, se tomó la pulpa y se desagregó en agua hasta formar una suspensión, la cual se pasó a través de un tamiz de 10 mesh (abertura de 2,00 mm) sobre el cual se recogieron las astillas residuales y por el que pasó la pulpa de nuevo a la bolsa de tela para su posterior centrifugación. Se ubicaron en el cuarto de condiciones controladas hasta alcanzar una humedad uniforme y luego realizar las pruebas. Se eliminó el exceso de humedad de las astillas residuales dejándolas al ambiente y luego se secaron en un horno a $103{ }^{\circ} \mathrm{C}$ hasta sequedad total y se determinó su masa seca. Con esta y con la masa seca final de la cocción se calculó el porcentaje de astillas remanentes.

Con los valores de las variables respuesta, se procedió a analizar estadísticamente los resultados y a obtener la significancia de la influencia de los efectos o de sus interacciones en los valores de las variables respuesta (o de estudio), determinando los valores en los que se maximizan o minimizan las variables respuesta según sea el caso. El análisis estadístico se realizó siguiendo el algoritmo y el procedimiento de cálculo y análisis presentado por Box, Hunter y Hunter (1978).

La pulpa obtenida a las condiciones más apropiadas se refinó en un refinador tipo pila holandesa, a una consistencia de 1,57\% en masa. Se tomaron alícuotas por duplicado a un mismo intervalo de tiempo para determinar la consistencia y el grado de refino, determinado indirectamente mediante la resistencia al drenaje Shopper-Riegler ( $\left.{ }^{\circ} \mathrm{SR}\right)$. Una vez alcanzado el grado de refino apropiado se registró el tiempo transcurrido en el proceso y se tomó una alícuota de la suspensión refinada para la formación de hojas de laboratorio, 
siguiendo la norma T-205 descrito en TAPPI (1996). Para el cálculo del rendimiento global, se sigue el procedimiento empleado por Gutiérrez y Blanco (1997), considerando los rendimientos parciales obtenidos en las operaciones de pulpeo, refinado y formación de hojas.

Los valores del grado de refino se corrigieron por masa convirtiendo el grado de resistencia al drenado real por medio de las curvas existentes para este fin o por medio de la matriz de datos que se brinda en TAPPI (1996). Estos resultados, junto con el tiempo de refino, se utilizaron para construir la curva de refino para la pulpa utilizada; además se determinó el valor de la masa seca final y con ella se calcula el rendimiento másico de la operación de refino.

Se formaron hojas de pulpa de laboratorio a partir de la mejor pulpa obtenida en el estudio, se acondicionaron a $23{ }^{\circ} \mathrm{C} \pm 2{ }^{\circ} \mathrm{C}$ de temperatura y $50 \% \pm 2 \%$ de humedad relativa y se caracterizaron siguiendo los procedimientos descritos por TAPPI (1996), todos los equipos de medición utilizados en este estudio están bajo control metrológico, por lo que se asegura la confiabilidad de los resultados.

\section{RESULTADOS}

\subsection{Características físicas de la madera y de las astillas}

En la Tectona grandis la diferencia entre albura y duramen es muy marcada, el duramen de color café oscuro (se encuentra en la parte interna o central del árbol) donde ocupa menos de la mitad del área total de la sección transversal del tronco. Los resultados de estas propiedades dependen mucho de la edad y de la procedencia de la muestra, por lo que los porcentajes de albura y duramen o el diámetro son muy propios de la madera estudiada, sus valores se presentan en el Cuadro 2.

Las características y dimensiones de las astillas utilizadas en el estudio se muestran en el Cuadro 3. La densidad experimentalmente obtenida fue de $0,45 \mathrm{~g} / \mathrm{cm}^{3}$ (desviación estándar de $0,01 \mathrm{~g} / \mathrm{m}^{3}$ ) es menor a la encontrada en la revisión bibliográfica para teca de bosque natural que es de $0,61 \mathrm{~g} / \mathrm{cm}^{3}$ y también al informado para teca de otras plantaciones. Esto se debe a que para esta investigación en particular, las astillas fueron obtenidas del tronco completo, con corteza inclusive, y siendo la corteza menos densa que la madera se obtiene un resultado menor.

Debido a que es casi imposible obtener astillas de tamaño homogéneo, se tamizaron para uniformizar, eliminando tanto las más grandes como las más pequeñas y el aserrín y polvo contenido en la muestra. A pesar de las variaciones en las dimensiones de las astillas, se observa que estas se encuentran cercanas a las dimensiones esperadas de $(25,4$ x 25,4) $\mathrm{mm}$. El fin de medir las dimensiones de las astillas es obtener el tamaño de partícula para el pulpeo, ya que esta es una de las variables de importancia en estudios de pulpeo, como se presentó en la revisión bibliográfica. Las astillas o "chips", como se conocen también en la industria, poseen forma de paralelepípedos, donde a pesar de los métodos de selección utilizados, se obtuvieron

Cuadro 2. Características físicas de la madera de Tectona grandis.

\begin{tabular}{lcc}
\multicolumn{1}{c}{ Parámetro } & Promedio & $\begin{array}{c}\text { Desviación } \\
\text { estándar }\end{array}$ \\
Humedad verde en base húmeda (\%) & 72,1 & 0,8 \\
Diámetro promedio total (cm) & 12,8 & 0,4 \\
Diámetro promedio duramen (cm) & 7,7 & 0,6 \\
Porcentaje área ocupado por duramen (\%) & 36,4 & --- \\
Porcentaje área ocupado por albura (\%) & 63,6 & ---
\end{tabular}

Fuente: (El autor y la autora). 
formas y tamaños muy variables. Las astillas escogidas poseían una humedad de $20,6 \%$ en base húmeda con una desviación estándar de $0,3 \%$; siendo suficientemente baja como para evitar su deterioro.

\subsection{Pulpeo a la soda antraquinona}

La pulpa obtenida era de color café claro, mucho más claro que las pulpas organosolventes; el color se fue aclarando durante el lavado, el contenido de astillas enteras después del pulpeo fue casi nulo, por lo que no se procedió a realizar la separación ni la cuantificación. En el Cuadro 4 se muestran los resultados que fueron obtenidos para los efectos e interacciones de las variables de respuesta para cada corrida, los mismos fueron calculados utilizando el algoritmo de Yates.

Para la variable de respuesta rendimiento másico de pulpa, todos los efectos (E1, E2, E3) se encuentran fuera del intervalo de no significancia, por lo que tienen una influencia significativa sobre el rendimiento de pulpa al pasar de un nivel a otro en cada variable.

El rendimiento, por el efecto de la variable temperatura (E1) disminuye en un 4,23\% al pasar de $160{ }^{\circ} \mathrm{C}$ a $170{ }^{\circ} \mathrm{C}$ del ámbito estudiado. Por lo tanto, como se quiere maximizar esta variable respuesta, se debe utilizar la temperatura en su nivel menor $\left(160{ }^{\circ} \mathrm{C}\right)$.
El efecto de la variable tiempo de cocción (E2) tiene el mismo comportamiento que el efecto de la temperatura, siendo el rendimiento másico disminuye en un 4,26\% en promedio, al pasar de su nivel menor al nivel superior. Igualmente, al buscarse el máximo posible de rendimiento, se debe trabajar la variable tiempo en su nivel menor (45 $\mathrm{min}$ ).

Por último, el efecto de la concentración de hidróxido de sodio (E3) tiende también a disminuir en promedio $2,51 \%$ el rendimiento al pasar de su nivel menor (7\%) a su nivel mayor (10\%); por lo tanto se determinó usar la concentración de hidróxido de sodio en $7 \%$, su valor menor.

Además de haber tres efectos significativos se presenta una interacción significativa, la interacción entre la concentración de hidróxido de sodio y el tiempo de cocción. Por medio del cuadro de interacción correspondiente, mostrado en la Figura 1, se puede observar que en cualesquiera de los casos, el pasar del valor menor al valor mayor de estas variables se produce una disminución en el rendimiento de pulpa, para el ámbito de las variables utilizado. Si se mantiene la variable tiempo de cocción en su nivel menor (45 $\mathrm{min}$ ), se produce la menor de las disminuciones como efecto sobre el rendimiento $(0,2 \%)$. Si permanece la concentración de hidróxido de sodio en su nivel menor (7\%) y pasa el tiempo de su nivel menor (45 min) a su nivel mayor

Cuadro 3. Características de las astillas de Tectona grandis.

\begin{tabular}{lcc}
\multicolumn{1}{c}{ Parámetro } & Promedio & Desviación estándar \\
Porcentaje de humedad $(\%)$ & 20,60 & 0,03 \\
Densidad aparente $\left(\mathrm{g} / \mathrm{cm}^{3}\right)$ & 0,45 & 0,01 \\
Largo promedio $(\mathrm{mm})$ & 26,29 & 3,10 \\
Largo mayor $(\mathrm{mm})$ & 36,43 & -- \\
Largo menor $(\mathrm{mm})$ & 20,94 & -- \\
Ancho promedio $(\mathrm{mm})$ & 21,51 & 2,59 \\
Ancho mayor $(\mathrm{mm})$ & 28,42 & -- \\
Ancho menor $(\mathrm{mm})$ & 16,39 & -- \\
Grosor promedio $(\mathrm{mm})$ & 3,95 & 0,84 \\
Grosor mayor $(\mathrm{mm})$ & 7,80 & -- \\
Grosor menor $(\mathrm{mm})$ & 2,39 & --
\end{tabular}

Fuente: (El autor y la autora). 
(75 min); se obtiene la segunda disminución más baja $(2,0 \%)$, por lo que después de analizar la interacción se refuerza lo dicho por los efectos por sí solos, utilizar ambas variables en su nivel menor.

Considerando la variable de respuesta contenida de lignina residual, después de aplicar el logaritmo de cálculo ninguno de los efectos o interacciones de las variables resultaron significativos, bajo el nivel de confianza establecido (90 \%) y dentro de los ámbitos utilizados para las variables; este mismo resultado fue encontrado por Rodríguez (2000), al utilizar el mismo proceso de pulpeo, lo que demuestra la buena selectividad y efectividad del pulpeo sodaantraquinona en la eliminación de la lignina del material utilizado, sea tanto en maderas como en especies no maderables como la paja de arroz. Los valores del contenido de lignina para cada corrida son muy similares, por lo que se sugiere más repeticiones que depuren la varianza estimada y sea más exacta y excluyente, así como ampliar o variar los límites de las variables utilizadas para evaluar mejor el efecto sobre la deslignificación.

Comparando ambos resultados, el ámbito establecido para las variables ofrece que para el rendimiento todas las variables son significativas y para el contenido de lignina ninguna lo es. Se

Cuadro 4. Valores de los efectos e interacciones para las variables del pulpeo a la soda antraquinona.

Valor
$\mu$
$\mathrm{E} 1$
$\mathrm{E} 2$
$\mathrm{I} 12$
$\mathrm{E} 3$
$\mathrm{I} 13$
$\mathrm{I} 23$
$\mathrm{I} 123$
Confianza $(\alpha)$
Grados de libertad $(\mathrm{V})$
Valor t-Student $\left(\mathrm{t}_{0}, 91,2\right)$
Varianza experimental $\left(\mathrm{S}_{E}^{2}\right)$
Intervalo no significancia
Into sia

Efectos e interacciones significativos

Rendimiento másico pulpa (\%)
40,8
$-4,227$
$-4,260$
0,925
$-2,510$
0,784
$-2,309$
0,848
0,10
2
1,889
0,802
$[-1,691 ; 1,691]$
E1, E2, E3, I23

Contenido de lignina residual (\% bps)

1,14

$-0,432$

$-0,101$

0,022

0,304

0,020

0,178

$-0,505$

0,10

2

1,889

0,252

$[-0,948 ; 0,948]$

Ninguno

Fuente: (El autor y la autora).

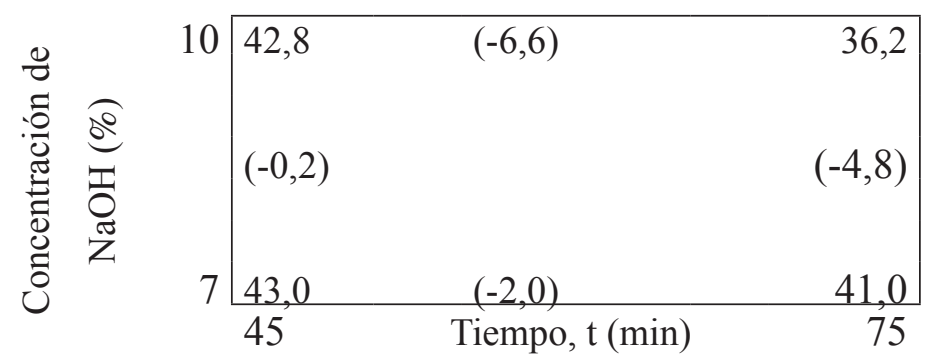

Figura 1. Cuadro de interacciones entre el tiempo y la concentración de $\mathrm{NaOH}$ para el rendimiento en el pulpeo de soda antraquinona. 
determina entonces que para el pulpeo sodaantraquinona, considerando ambas variables de respuesta, es mejor utilizar las variables en sus valores menores: la temperatura $\left(160{ }^{\circ} \mathrm{C}\right)$, el tiempo de cocción (45 min) y la concentración de hidróxido de sodio (7\%). En comparación con otros procesos de pulpeo y otras materias primas, este pulpeo es bastante efectivo, principalmente en lo relativo al tiempo, es difícil observar pulpeos que en menos de una hora logren tan buenos resultados. De las corridas realizadas, la corrida número 1 es la que corresponde a estas condiciones, se obtuvo $45,5 \%$ de rendimiento másico de pulpa y $1,61 \%$ de contenido de lignina residual. El rendimiento fue el más alto en todas las corridas, el contenido de lignina no es el menor, pero en general todos los resultados son muy cercanos.

En el pulpeo soda-antraquinona, es de esperar que se eliminen los solubles en agua caliente y en hidróxido de sodio (alrededor de un $13 \%$ ) más el $30 \%$ de lignina, en total, como máximo un 43\%; lo que representaría un rendimiento máximo (de únicamente holocelulosa) de $57 \%$, sin embargo, se obtuvo un rendimiento máximo del $46 \%$, o sea parte de la celulosa se eliminó. En este caso, el contenido de solubles en hidróxido de sodio debe ser mucho mayor que el obtenido en la caracterización química, por lo que el material eliminado no celulósico es mayor al antes propuesto, lo que indica una menor eliminación de celulosa y por ende, un mejor desempeño del proceso de pulpeo.

Dado que la mayoría de las maderas presentan composiciones químicas muy similares, en la literatura técnica relativa se informa que los procesos de pulpeo químico tienen un rendimiento aproximadamente del $50 \%$, por lo que, el rendimiento obtenido es muy aceptable, considerando que las pérdidas de celulosa no solo se dan por las reacciones químicas durante el pulpeo, sino también por pérdidas en los lavados y pérdida de paredes de las fibras. Se recomienda para otros experimentos determinar el contenido de celulosa antes del pulpeo y posterior al pulpeo y determinar un rendimiento más exacto que busque una evaluación más estricta del pulpeo soda-antraquinona y no tanto de la materia prima.

\subsection{Escogencia de la mejor corrida}

A partir de los resultados de los análisis estadísticos, se determinó que la mejor pulpa del pulpeo soda-antraquinona se obtuvo con $7 \%$ de hidróxido de sodio, $160{ }^{\circ} \mathrm{C}$ de temperatura y 45 min de cocción, obteniéndose un 45,5\% de rendimiento másico y un 1,61\% de lignina residual. El rendimiento de pulpeo no es un parámetro definitivo y es cercano al $50 \%$ que plantea la teoría para pulpeos químicos.

Las astillas remanentes después del pulpeo se encontraban en tan poca cantidad (casi inapreciables) que no se cuantificaron. La presencia de astillas, se prefiere baja o cero pues facilita el posterior refino y en sí la calidad de la pulpa final. La deslignificación es otro parámetro por analizar, en este caso el bajo contenido de lignina en la pulpa define una deslignificación alta que sigue favoreciendo este pulpeo.

Se utilizaron $450 \mathrm{~g}$ de astillas secas con los cuales se realizaron dos pulpeos de $225 \mathrm{~g}$ cada uno, utilizando una temperatura de $160{ }^{\circ} \mathrm{C}$ una concentración de hidróxido de sodio de $7 \%$ y un tiempo de cocción de $45 \mathrm{~min}$, manteniendo la relación sólido a líquido en 1:6 y la concentración de antraquinona en $0,1 \%$.

Es importante mencionar la reproducibilidad de los resultados obtenidos en el diseño estadístico, al menos para la corrida en cuestión. Se realizaron las dos corridas obteniéndose al final de cada una la misma cantidad de pulpa seca, 101,3 g, lo que representa un rendimiento de $45,0 \%$ en las dos muestras, resultado prácticamente igual al obtenido en el diseño estadístico.

La pulpa obtenida estaba libre de astillas visibles a simple vista y presentaba el mismo color que la corrida original color beige. Esta pulpa se ajustó con la pulpa de la corrida original para obtener $240 \mathrm{~g}$ de pulpa seca que se utilizaron en el refino. Se midió inicialmente el punto cero y se obtuvo un grado de resistencia al drenaje inicial de $13,5{ }^{\circ} \mathrm{SRc}$, un valor bajo comparado con los reportados por Gutiérrez (1990) para otras maderas, como eucalipto y melina, las cuales para diferentes condiciones y procesos presentan valores iniciales entre $(30 \mathrm{y}$ $18)^{\circ} \mathrm{SR}$. Esto puede representar problemas en un 
eventual proceso industrial por la energía que se deba emplear para llevar el material a un grado de refino deseado, pero indica en sí las condiciones que tiene la pulpa y no tanto las características o el comportamiento que tengan las fibras.

\subsection{Proceso de refino}

Se realizó el refinado de la pulpa en un refinador de pulpa tipo pila holandesa. Se dejó la pulpa desde el día anterior en remojo para facilitar su desagregación y se trabajó a una consistencia de 1,6\%. Se dejó agitando la pulpa sin aplicar carga (sin refinar) para su buen remojo. Previo al refino de la pulpa, se realizó, a manera de prueba, el refinado de una pulpa correspondiente a una corrida muy semejante a la preparada (según los resultados del diseño estadístico) para así observar el comportamiento de la pulpa, evaluar su facilidad o dificultad de refino, los pesos necesarios para el refino, tiempos adecuados para la toma de alícuotas para medir la resistencia al drenaje, entre algunos de los factores analizados. La importancia de dicha prueba fue, principalmente, evitar refinar en exceso la pulpa y en general obtener la mayor y mejor información posible del refino sin emplear más tiempo del necesario. Se observó que la pulpa no presenta dificultades de refino, es decir, a pesar de tener un valor inicial tan bajo de resistencia al drenaje, es posible en poco tiempo llegar a valores altos, incluso de $50{ }^{\circ} \mathrm{SR}$ o de $70{ }^{\circ} \mathrm{SR}$ en poco más de una hora. Se utilizaron pesas que en total sumaron $10 \mathrm{~kg}$, como carga aplicada sobre el brazo de la pila con la cual se da la fuerza para refinar la fibra. Tomando en cuenta las consideraciones derivadas de la etapa de prueba se realizó el refino de la mejor pulpa y con los datos obtenidos para esta nueva etapa de refino se construyó la curva, mostrada en la Figura 2.

Analizando la curva de refino, se observa un comportamiento creciente en todo instante analizado, iniciando en el valor de $13,5{ }^{\circ} \mathrm{SRc}$, y finalizando en un valor cercano a $45^{\circ} \mathrm{SRc}$. Se tomó la alícuota para formación de hojas a los
33 min de refino porque a ese tiempo presentaba un grado de refino cercano a $41^{\circ} \mathrm{SR}$, adecuado para la formación de hojas de laboratorio. La curva presenta el comportamiento esperado, una tendencia creciente al principio, llegando a un intervalo de tiempo donde se mantiene casi constante. Si se continuara refinado se esperaría un decrecimiento por un deterioro excesivo de las fibras. Como se logró comprobar a través de la etapa de prueba, las fibras aun a $70{ }^{\circ} \mathrm{SR}$ muestran el comportamiento creciente, así que la curva se suspendió en un valor mucho menor ya que el máximo se encuentra muy por encima de las condiciones de refino deseadas como para estudiarlo en la misma curva.

El tiempo empleado en llegar al drenado deseado es en realidad un tiempo bastante corto si se compara con curvas de refino para otras pulpas; como por ejemplo, para el caso de una pulpa mecánica de melina, Ruiz (2000) obtuvo que en 300 min la pulpa no alcanza ni siquiera $15^{\circ} \mathrm{SR}$, o bien el caso de la pulpa de paja de arroz estudiada por Rodríguez (2000) que iniciando cerca de $37{ }^{\circ} \mathrm{SR}$, necesitó de casi una hora para obtener el mismo grado de drenado obtenido para la pulpa de teca al momento de tomar la alícuota para formación de hojas. Esto comprueba la facilidad que presenta la pulpa de teca al refino, siendo esta una característica de enorme importancia considerando el consumo energético durante el refino de pulpas. Se puede destacar además el buen desempeño del pulpeo químico, ya que el tiempo de refino es sustancialmente menor al obtenido en el refino de la pulpa mecánica de melina, que es una especie de excelentes características para fabricación de papel. Además, es importante considerar el hecho de que el refinador utilizado no es el más eficiente; a nivel de laboratorio existen otros mejores y aún más eficientes a nivel industrial, mostrando que sería posible obtener la pulpa con el grado de refino deseado en un tiempo realmente corto, para la clase de operación que se está llevando a cabo.

Las propiedades obtenidas de las hojas de pulpa de laboratorio después del pulpeo, se muestran en el Cuadro 5. 


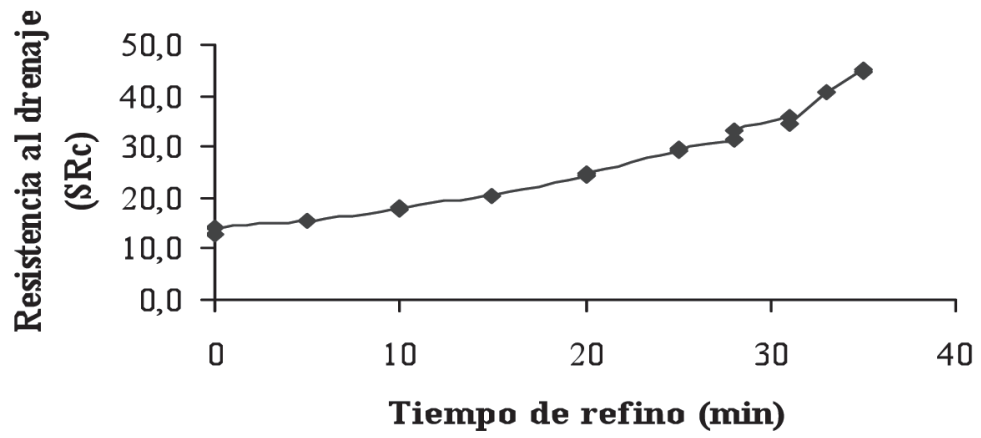

Figura 2. Curva de refino en la pila holandesa de la mejor pulpa obtenida con el pulpeo soda antraquinona.

Fuente: (Abarca, 2003).

\section{CONCLUSIONES}

1. La madera de Tectona grandis de seis años proveniente de la plantación de Aguas Zarcas de San Carlos, presentó una humedad verde de $72,1 \%$ en base húmeda, un diámetro total promedio de $12,8 \mathrm{~cm}$ con $7,7 \mathrm{~cm}$ de diámetro en promedio de duramen, ocupando este un $36,4 \%$ del área superficial de la sección transversal del tronco. Las astillas tenían una humedad de 20,6\% en base húmeda y una densidad de $0,45 \mathrm{~g} / \mathrm{cm}^{3}$. Las dimensiones de las astillas fueron en promedio $26,3 \mathrm{~mm}$ de largo con una desviación de 3,1 mm; 21,5 $\mathrm{mm}$ de ancho con una desviación estándar de 2,6 $\mathrm{mm}$ y $4,0 \mathrm{~mm}$ de espesor con una desviación de $0,8 \mathrm{~mm}$.

2. Se obtuvo la mejor pulpa soda-antraquinona utilizando las condiciones menos drásticas estudiadas en los experimentos: el tiempo más corto y la temperatura más baja, a saber $160{ }^{\circ} \mathrm{C}, 45 \mathrm{~min}$, además y una concentración de hidróxido de sodio del $7 \%$. El rendimiento másico de pulpa fue excelente, un 45,5\% y el contenido residual de lignina de 1,6\% (deslignificación de $98 \%$ ). Se obtiene una pulpa de color beige, claro y sin astillas remanentes después del tratamiento. El rendimiento másico es comparable para pulpeos químicos de especies maderables y cercanas al $50 \%$.

3. El pulpeo soda-antraquinona presentó una excelente efectividad en cuanto al procesamiento y cocción de la madera para la producción de pulpa, siendo muy selectiva en la eliminación de la lignina sin deterioro de la celulosa, lo que se reflejó en un rendimiento alto, para una etapa de laboratorio en condiciones que pueden mejorarse a nivel industrial. El tiempo fue más corto que el empleado en otras investigaciones similares, y la temperatura y la concentración de hidróxido de sodio a pesar de ser bajas podrían disminuirse de acuerdo con los resultados del experimento.

4. En promedio las hojas de pulpa sodaantraquinona presentaron una densidad aparente mediana $\left(600 \mathrm{~kg} / \mathrm{m}^{3}\right)$, baja permeabilidad al aire $(23,3 \mathrm{~s} / 100 \mathrm{~mL})$, $\mathrm{pH}$ neutro $(6,83)$, bajo contenido de cenizas, opacidad muy alta (más de 97 $\%)$ y baja brillantez (30,6 \%). Además sus propiedades mecánicas fueron baja resistencia a la explosión $(1,8 \mathrm{kN} / \mathrm{g})$, de mediana a baja longitud de ruptura $(3,18$ $\mathrm{km})$, baja resistencia a la tensión $(31,2$ $\mathrm{N} \cdot \mathrm{m} / \mathrm{g}$ ) y un valor medio de resistencia al rasgado $\left(3,9 \mathrm{mN} \cdot \mathrm{m}^{2} / \mathrm{g}\right)$. El color es café claro $(\mathrm{L}=71,4 ; \mathrm{a}=5,8 ; \quad \mathrm{b}=15,6)$ y no presentan puntos negros. Las hojas presentan alta uniformidad, facilidad de formación, ausencia de materiales extraños como residuos de madera, puntos negros y en general presentan características (físicas, mecánicas, químicas y ópticas) similares y en la mayoría de casos mejores que otras pulpas obtenidas de especies maderables bajo procesos de pulpeo similares.

5. La pulpa soda-antraquinona de tectona grandis se puede utilizar para la fabricación de papeles 
Cuadro 5. Propiedades de las hojas de pulpa soda antraquinona de Tectona grandis, refinadas en pila holandesa a $41{ }^{\circ} \mathrm{SRc}$ y medidas a $50 \% \pm 2 \%$ y $23{ }^{\circ} \mathrm{C} \pm 2{ }^{\circ} \mathrm{C}$.

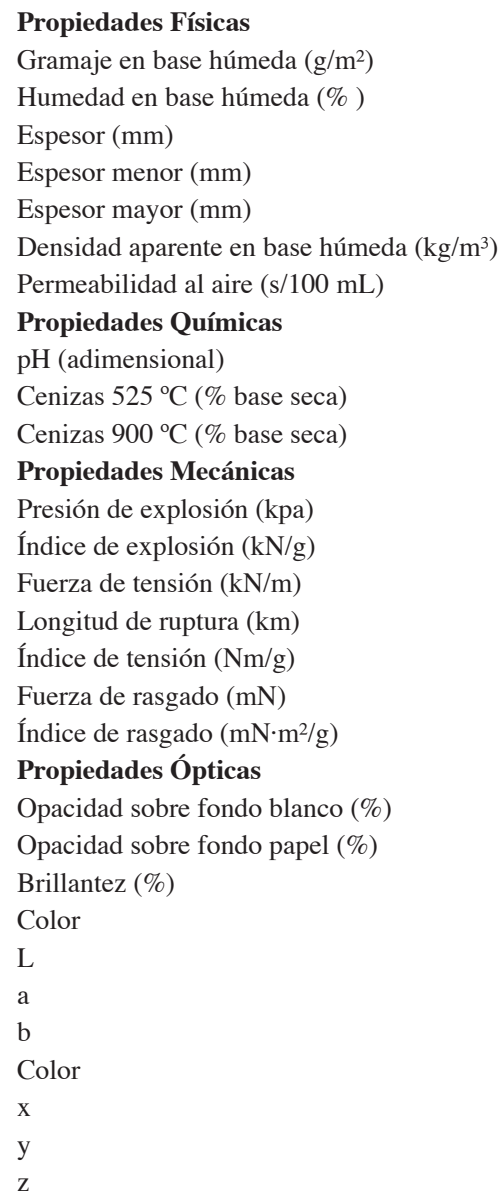

Valor promedio

$\begin{array}{cc}62,2 & 0,15 \\ 5,72 & 0,15 \\ 0,104 & 0,005 \\ 0,091 & - \\ 0,137 & - \\ 600 & 55 \\ 23,3 & 2,6 \\ & \\ 6,83 & 0,07 \\ 1,49 & 0,05 \\ 1,37 & 0,04 \\ & \\ 114,3 & 4,6 \\ 1,8 & 0,1 \\ 1,94 & 0,11 \\ 3,18 & 0,18 \\ 31,2 & 1,8 \\ 245,3 & 19,6 \\ 3,9 & 0,3 \\ & \\ 97,6 & 3,8 \\ 100,5 & 2,6 \\ 30,6 & 0,3 \\ & 0,3 \\ 71,41 & 0,05 \\ 5,78 & 0,03 \\ 15,56 & 0,07 \\ 46,27 & \\ 42,78 & 0,08 \\ 21,31 & \\ & \\ & \\ & \\ & \\ & \\ & \\ & \end{array}$

Fuente: (Abarca, 2003).

para impresión, bond, sobres, cuadernos; utilizando una etapa de blanqueo posterior o en empaques de baja resistencia, paneles de construcción y aglomerados; o en papeles de mayor calidad mezclando con pulpas de fibras más largas, entre otras aplicaciones.

Por último, se recomienda realizar más repeticiones de las corridas del diseño estadístico del pulpeo soda-antraquinona para depurar y hacer más estricta la varianza experimental y poder obtener más información del efecto de las variables con respecto a la lignina residual y ampliar el estudio utilizando condiciones menos drásticas. Además, se recomienda estudiar la teca bajo el pulpeo a la soda y determinar efectivamente el efecto de la antraquinona sobre las características de la pulpa.

También, se podría realizar un análisis de $\alpha$ -celulosa antes y después del pulpeo para definir mejor la selectividad en la separación de ligninas.

\section{REFERENCIAS BIBLIOGRÁFICAS}


Abarca, R. (2003). Caracterización de Tectona grandis de plantación y evaluación del uso potencial de la pulpa y la lignina obtenidas. Proyecto de graduación para optar al grado de Licenciatura en Ingeniería Química, Escuela de Ingeniería Química, Universidad de Costa Rica, San José, Costa Rica. p 177.

Gutiérrez, J. R. \& Blanco, M. L. (1997). Evaluación de los tallos de caña india (Dracaena massangeana) e itabo (Yucca elephantipes) para la producción de pulpa para papel. Laboratorio de Productos Forestales, Instituto de Investigaciones en Ingeniería, Universidad de Costa Rica.

Box, G., Hunter, W. \& Hunter, J. (1998). Statistics for experiments. USA: Jonh Willer \& Sons, Inc.

Casey, J. (1990). Pulpa y papel: química y tecnología química ( $1^{\mathrm{a}}$ ed. en español) (Vol. 1). México: LIMUSA.

Gomes da Silva, F. (1994). Conversão do processo kraft em soda-DDA (sal disódico de 1,4-dihidro-9,10-dihidroxiantraceno) para madeira de eucalipto. São Paulo, Brasil.

Gómez, J. A. (1980). Obtención de pulpa para la fabricación de papel, usando etanol acuoso como agente de pulpeo. Proyecto de graduación para optar por el grado de Licenciatura en Ingeniería Química, Escuela de Ingeniería Química, Universidad de Costa Rica, San José, Costa Rica.

Gutiérrez, J. R. (1990). Características del pulpeo organosolvente modificado de Eucalyptus saligna. Proyecto de graduación para optar por el grado de Licenciatura en Ingeniería Química, Escuela de Ingeniería Química, Universidad de Costa Rica, San José, Costa Rica.

LATU. (1992). Manual de técnicas de ensayo de pulpa de celulosa y papeles. Laboratorio Tecnológico del Uruguay, Uruguay.
Manji, A. (1996). Kraft pulping of coastal softwood from British Columbia using AQ and a digester additive. TAPPI Journal, United States.

Rodríguez, J. (2000). Características de la paja de arroz (Oriza sativa) y evaluación de la pulpa obtenida para la producción de papel. Proyecto de graduación para optar por el grado de Licenciatura en Ingeniería Química, Escuela de Ingeniería Química, Universidad de Costa Rica, San José, Costa Rica.

Ruiz, J. (2000). Pulpeo biológico de Gmelina arborea utilizando el hongo Trametes villosa. Proyecto de graduación para optar por el grado de Licenciatura en Ingeniería Química, Escuela de Ingeniería Química, Universidad de Costa Rica, San José, Costa Rica.

TAPPI. (1996). TAPPI Test Methods. TAPPI, Technical Association of the Pulp and Paper Industry, TAPPI Press, Atlanta.

\section{SOBRE LOS AUTORES}

\section{Ricardo Abarca Espeleta}

Licenciado en Ingeniería Química de la Universidad de Costa Rica

Teléfono: (506) 22026163

Facsímil: (506) 22534614

Correo electrónico: ricardo.abarca@ costarricense.cr

\section{María Lorena Blanco Rojas}

Licenciada en Ingeniería Química de la Universidad de Costa Rica

Magister Scientiae en Ciencia y Tecnología de la Madera de la Universidad de São Paulo, Brasil

Profesora catedrática de la Universidad de Costa Rica

Investigadora del Instituto de Investigaciones en Ingeniería

Teléfono: (506) 25116163

Facsímil: (506) 25114614

Correo electrónico: lorena@inii.ucr.ac.cr 